\title{
Multi-layer structure of mid-latitude sporadic- $E$ observed during the SEEK-2 campaign
}

\author{
M. Wakabayashi and T. Ono \\ Graduate School of Science, Tohoku University, Sendai, Miyagi, Japan \\ Received: 15 December 2004 - Revised: 28 June 2005 - Accepted: 26 August 2005 - Published: 13 October 2005 \\ Part of Special Issue "SEEK-2 (Sporadic-E Experiment over Kyushu 2)"
}

\begin{abstract}
In the mid-latitude ionospheric region, sporadic- $E$ layers ( $E_{s}$ layers) have often been observed, revealing multiple layers. The $E_{s}$ layers observed during the SEEK-2 rocket campaign showed double electron density peaks; namely, there are stable lower peaks and relatively unstable upper peaks. We examined the effects of wind shear and the electric fields on the generation of the multiple layer structure, in comparison with the electron density profile, the neutral wind, and the DC electric field observed by the $S 310$ rocket experiments. The results showed that the neutral wind shear is mainly responsible for the generation of the lower layer, while the DC electric field makes a significant contribution to the formation of the upper layer. The difference between the lower and upper layers was also explained by the enhanced AC electric field observed at about $103-105 \mathrm{~km}$ altitude. The external DC electric field intensity is expected to be $\sim 5 \mathrm{mV} / \mathrm{m}$, which is enough to contribute to generate the $E_{s}$ layers in the ionosphere.
\end{abstract}

Keywords. Ionosphere (Electric fields; Ionospheric irregularities, Mid-latitude ionosphere)

\section{Introduction}

Since the first identification in the 1930's, the mid-latitude $E_{s}$ layer has a long history of research which extends over 60 years. Many observations have been carried out by using ionosondes and radars (e.g. Smith and Wright, 1972; Ogawa et al., 2002; Maruyama et al., 2003; Haldoupis et al., 2003), and rocket experiments (e.g. Dorling et al., 1969; Yamamoto et al., 1998; Roddy et al., 2004). The generation mechanism of the mid-latitude $E_{S}$ layer has been understood using the wind shear theory (e.g. Whitehead, 1961; Axford, 1963) and many observation results have supported this interpretation (e.g. Fujitaka and Tohmatsu, 1973; Kato et al., 1972; Yamamoto et al., 1998); namely, there is good correspondence

Correspondence to: M. Wakabayashi

(waka@stpp1.geophys.tohoku.ac.jp) between the observed $E_{S}$ layer altitude (the altitude of electron density peak) and the predicted altitude (wind shear altitude) derived from the neutral wind profile. Moreover, the ionospheric electric field has also been found to contribute to the dynamics of the mid-latitude $E_{S}$ layer, while the classical wind shear theory ignored any external ionospheric electric field. For example, Rees et al. (1976) reported that the electric field effect helped to stabilize the $E_{S}$ layer structure which was formed by the wind shear mechanism. Based on Incoherent Scatter Radar (ISR) observations over Arecibo, Mathews et al. (2001) suggested that the plasma instability in the $E_{S}$ layer could be triggered by the electric fields penetrating from the $F$-region. The layer formation under the effects of wind shear and the electric field was investigated with the 3-D simulation by Machuga and Mathews (2001). It was suggested that the horizontal electric field was able to modify the altitude and formation of the $E_{s}$ layer.

Huuskonen et al. (1988) pointed out the similarities and differences of $E_{s}$ layers observed in the mid-latitude and polar regions in terms of appearance and ion composition. They showed that different generation mechanisms of the $E_{s}$ layer are necessary because the magnetic dip angle in the auroral region is larger than at mid-latitudes. The wind shear mechanism doesn't work well at high latitudes because the horizontal component of the magnetic field is too weak to accumulate the ions via $\boldsymbol{V} \times \boldsymbol{B}$ drift (Kirkwood and von Zahn, 1991). In the polar region, electric field effects are important to the formation of the $E_{S}$ layer, as demonstrated by $\mathrm{Ny}$ gren et al. (1984). Kirkwood and von Zahn (1991) showed computer simulations demonstrating the formation of the $E_{s}$ layer by electric fields in the absence of neutral wind effects. Based on EISCAT radar observations, Kirkwood (1997) reported that the height distribution and local time dependence of the $E_{s}$ layers are controlled by the combined effects of tidal wind and the electric field.

However, at mid-latitudes, the seasonal dependence (the maximum in summer) and steep horizontal gradient of density profiles of the $E_{S}$ layers are not yet understood. In addition, neutral sodium (or Fe) layers are often observed 
accompanying the $E_{s}$ layer (Kirkwood and von Zahn, 1993). Kirkwood and von Zahn (1993) pointed out that such a neutral metal layer would be ionized by auroral particle precipitation, and it would be observed as a multiple $E_{s}$ layer when it was accompanied by an ordinary $E_{S}$ layer in the polar region. On the other hand, in the mid-latitude region, the present theory is insufficient because the wind shear mechanism does not explain such a dense and narrow layer with the density peaks of $10^{5} \mathrm{~cm}^{-3}$ in the background ionization of $10^{3} \mathrm{~cm}^{-3}$ (Yokoyama, private communication, 2005). The numerical simulations by Yokoyama et al. (private communication, 2005) showed that the synthesized $E_{s}$ layer is too stable to describe the behavior of the actual $E_{s}$ layer. Thus, other physical processes, such as chemical reactions or anomalous ionization are needed to understand the real behavior of the $E_{s}$ layer.

Complex $E_{S}$ structures have often been observed using radar (Mathews et al., 1993) and rocket observations (Bowen et al., 1964; Smith, 1966; Smith and Mechtly, 1972; Kato et al., 1972). During the SEEK rocket campaign, such structures were also observed (Yamamoto et al., 1998). The double peaked $E_{s}$ structure measured by Smith and Miller (1980) was explained by the unstable wind shear; however, their interpretation has not been verified by simultaneous and direct observations of electron density and neutral wind. The ISR observations over the Arecibo observatory also revealed complex $E_{s}$ structures; for example, Mathews et al. (2001) observed the $E_{s}$ modulation accompanied by $F$ region disturbance. They argued that such an $E_{s}$ structure is due to the ionospheric electric field which originates from $F$ region disturbances. In addition, different ion compositions were observed based on the ISR observations of the $E_{s}$ layers (Huuskonen et al., 1988). However, it is noted that all the $E_{s}$ layers having the multiple structure are not always composed of different ion species.

In recent years, computer simulation results have suggested that plasma instabilities could deform the $E_{s}$ layer into a complex structure. Bernhardt (2002) proposed that the Kelvin-Helmholtz Instability (KHI) could generate the double $E_{s}$ layer centered on the wind shear altitude. Cosgrove and Tsunoda (2003) also suggested that another type of nonlinear instability could generate a complex layer structure. They concluded that the instability could lift up part of the $E_{S}$ layer to overlap with the original layer. Therefore, an external electromagnetic force becomes an important candidate for the formation and dynamics of the $E_{s}$ layer.

Based on the above background, the SEEK-2 rocket campaign was carried out on 3 August 2002, in order to observe the $E_{S}$ layer related to the QPEs (Yamamoto et al., 2005). As a result of the SEEK-2 experiment, $E_{s}$ double layer structures were observed with steep density gradients at altitude locations of around $100 \mathrm{~km}$, with altitude intervals of $1 \mathrm{~km}$. During the rocket experiments, the strong wind shear was also observed at the same altitude as the $E_{s}$ layer peaks. The purpose of this paper is to evaluate the wind shear theory for explaining the complex $E_{s}$ layer observed during the SEEK2 campaign. The second purpose is to evaluate the electric field effects. We investigate the generation mechanisms by analyzing the difference between observed layers for ascending and descending phases of two rocket trajectories.

\section{$2 E_{s}$ layer observation during the SEEK-2 campaign}

SEEK-2 is the comprehensive observation campaign in the mid-latitude ionosphere employing two sounding rockets (S310-31, -32) and ground-based instruments (VHF radar, ionosonde, etc.). The purpose of the campaign was to reveal the generation mechanism of QPEs and its relationship to the $E_{s}$ layer. It was carried out on 3 August 2002 at Kyushu Island, Japan. As planned, two rockets were launched with a time separation of $15 \mathrm{~min}$. The successive launches made it possible to obtain the structure and dynamics of the $E_{s}$ layer associated with the QPE phenomena (this is one of the main advances over the previous SEEK campaign (Fukao et al., 1998)). In the present analysis, we examine the electron density data (NEI instrument) (Wakabayashi et al., 2005), the DC electric field (EFD instrument) (Pfaff et al., 2005), the neutral wind velocity (TMA) (Larsen et al., 2005) and the AC electric field (PWM instrument) (Wakabayashi et al., 2005). Electron density was measured using the S310-31, and S310-32 rockets. The electric field was measured on board S310-31, while wind velocity and the AC electric field was measured on board S310-32. The AC electric measurement was planned to observe the plasma waves associated with the TMA release.

Neutral wind and electron density data have an altitude resolution of $1 \mathrm{~km}$ and about $400 \mathrm{~m}$, respectively. Plasma waves were measured within the frequency range below $10 \mathrm{kHz}$; however, in this paper we use the waveform data obtained below $100 \mathrm{~Hz}$.

\section{Observations and analysis}

\subsection{Electron density measurement}

Figure 1 shows the electron density profiles of the $E_{s}$ layers obtained by using both rockets during ascending and descending phases (within the altitude range from 95 to $110 \mathrm{~km})$. These profiles indicate that the $E_{s}$ layers appeared at almost the same altitude range, revealing one or two sharp peaks. These peaks are analyzed with respect to the density, altitude and thickness, and compared with other observations of the electric field, neutral wind, and plasma wave measurements.

The plasma density data show, that the lower peaks (indicated as $31-1,31-1^{\prime}, 32-1$, and $32-1^{\prime}$ in Figs. $1 \mathrm{a}$ and b) in the altitude range from 101.4 to $102.7 \mathrm{~km}$ were a stable feature, with a small variation in the density value within a factor of 1.9 among the four paths of the two rockets. However, the upper layers (indicated as 31-2, 31-2', 32-2, and $32-2^{\prime}$ in Figs. 1a and b) in the range from 103.2 to $105.0 \mathrm{~km}$ exhibit greater variability in their density, as well as the location height among the four paths. The upper layer observed 
in the S310-31 descending phase (31-2') had nearly the background density value. On the other hand, data of the S310-32 ascending phase (32-2) had the density peak giving the maximum value during the two rockets flights. And their peak altitudes are in ranges of 1.3 and $1.8 \mathrm{~km}$ for the lower (31-1, $31-1^{\prime}, 32-1$, and 32-1') and upper (31-2, 31-2', 32-2, and 32$\left.2^{\prime}\right)$ layers, respectively. So, the higher layers tend to appear in a slightly broad altitude range. In addition, when considering their shapes, the thicknesses (vertical distance between the upper and lower boundary of each layer) of the lower and upper layers are ranging from 1.4 to $2.9 \mathrm{~km}$ and from 1.4 to $1.9 \mathrm{~km}$, respectively. Therefore, the upper layers tend to appear as the relatively narrow and sharp peaks. In summary, the lower layers are located at the similar altitudes with stable densities and wide thickness. On the other hand, the upper layer shows the variable nature in their location altitude, shape and density.

\subsection{Comparison with wind shear theory}

The vertical profiles of the electron number density measurements are compared with the results of neutral wind profiles observed by the TMA release experiment. Because the TMA release was carried out for ascending and descending phases of the S310-32 experiment (Larsen et al., 2005), we try to compare the measured electron number density and the wind profiles with the altitude intervals of $1 \mathrm{~km}$. Based on the wind data, the vertical ion drift velocity, due to the wind shear, is derived by adopting the method of MacLeod (1966). After MacLeod (1966), the following equations are applied to the observation results, namely, the equation of motion is modified by assuming the equilibrium state, as

$\rho_{+}\left(\boldsymbol{u}-\boldsymbol{v}_{i}\right)+\boldsymbol{v}_{i} \times \boldsymbol{b}=0$,

where

$\rho_{+}=\frac{m_{i} v_{n i}}{e_{i} B}=\frac{v_{n i}}{\omega_{i}}$,

$\boldsymbol{B}=B \boldsymbol{b}$,

where $\boldsymbol{b}$ is unit vector in the direction of the magnetic field, and $\boldsymbol{u}$ and $\boldsymbol{v}_{i}$ are the neutral wind velocity, and the velocity of ion motion, respectively.

Equation (1) is derived from the equation of motion for a single particle, neglecting the pressure and the effect of the external electric field. The $z$ component of ion velocity $\boldsymbol{V}_{i}$ can be obtained,

$\boldsymbol{V}_{i}=\frac{1}{1+\rho_{+}^{2}}\left[\rho_{+}^{2} \boldsymbol{u}+\rho_{+} \boldsymbol{u} \times \boldsymbol{b}+(\boldsymbol{u} \cdot \boldsymbol{b}) \boldsymbol{b}\right]$.

For the present analysis, the geomagnetic dip and the declination are given by the IGRF geomagnetic field model. The collision frequency between ions and neutral particles is given by the relation of

$v_{\text {in }} \approx 5 \times 10^{-10} N_{n}[\mathrm{~Hz}]$,

where $N_{n}$ indicates the neutral particle density $\left(\mathrm{cm}^{-3}\right)$.
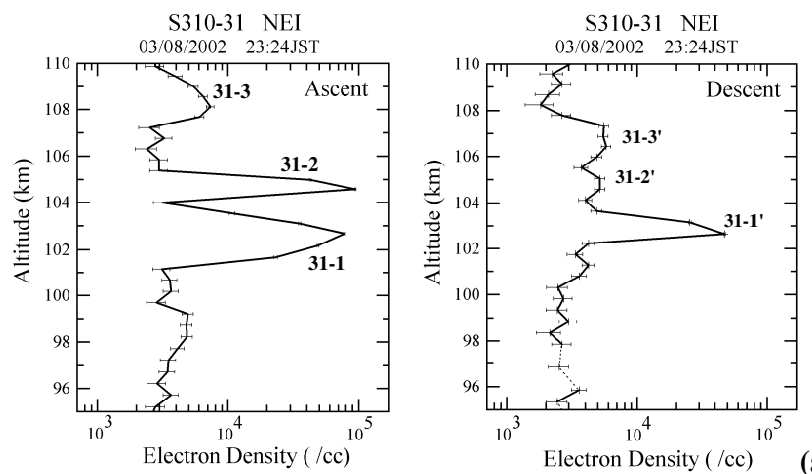

(a)
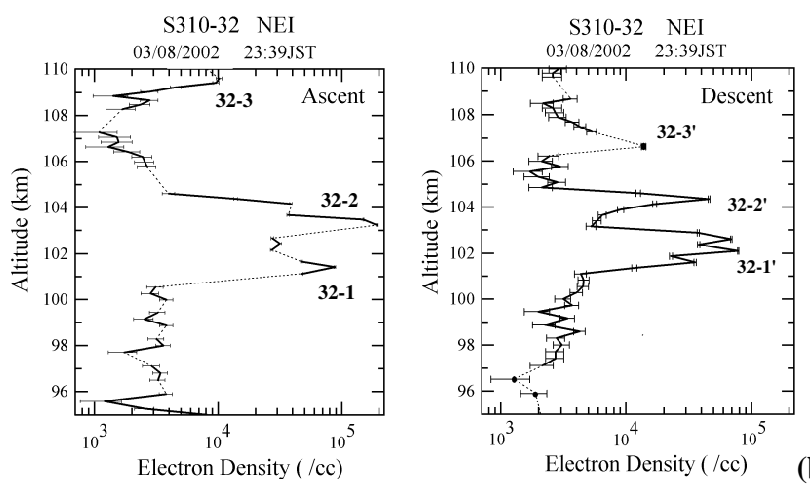

(b)

Fig. 1. (a) Altitude profiles of electron number density obtained using the NEI instrument on board the S310-31 rocket (these panels represent the altitude range from 95 to $110 \mathrm{~km}$ of Fig. 4a in Wakabayashi et al. (2005)). There are sharp peaks due to $E_{S}$ layers at the altitude of about 102.7 and $104.6 \mathrm{~km}$ during the ascending phase (peaks are numbered as $31-1$ and $31-2$ ). There is only one sharp peak during the descending phase at the altitude of $102.6 \mathrm{~km}$ (this peak is indicated as $31-1^{\prime}$ ). (b) Altitude profiles of electron number density obtained using the NEI instrument on board the S31032 rocket. Several dashed lines indicate the data blanks due to the TMA release. There are three enhancements of density at altitudes of 101.4, 103.2 and $109.6 \mathrm{~km}$ during the ascent (they are numbered as 32-1, 32-2 and 32-3, respectively). The peaks during the descent are also numbered as well as during the ascent.

The ion cyclotron frequency was approximated as

$\omega_{i} \approx 1 \times 10^{2}[\mathrm{~Hz}]$.

In Figs. 2a and $\mathrm{b}$, the $V_{z}$ profiles and electron density profiles are compared directly. It is shown that there are three possible altitude ranges where ions are accumulated as a layer during the ascending period (gray shadings in Fig. 2a), namely, they are located from $100 \mathrm{~km}$ to $103 \mathrm{~km}$ (particularly at $102-103 \mathrm{~km}$ ), from $106 \mathrm{~km}$ to $108 \mathrm{~km}$ (particularly at $106-$ $107 \mathrm{~km}$ ), and from $109 \mathrm{~km}$ to $110 \mathrm{~km}$. In comparison with the electron density measurement on board S310-32, we can confirm corresponding peaks at altitudes of $101.4 \mathrm{~km} \mathrm{(32-1)}$ and $109.6 \mathrm{~km}$ (32-3). However, 32-2 did not correspond to a strong ion accumulation rate.

On the other hand, in the descending phase of S310-32, the $V_{z}$ profile also suggests that there are three altitude regions where the ion accumulation occurs, namely they are located from $96 \mathrm{~km}$ to $98 \mathrm{~km}$, from $100 \mathrm{~km}$ to $103 \mathrm{~km}$, and from 
(a)

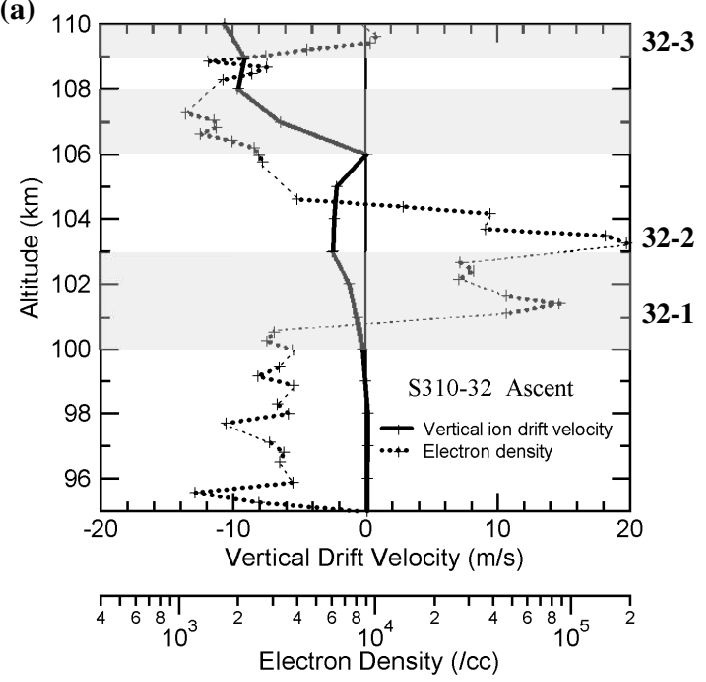

(b)

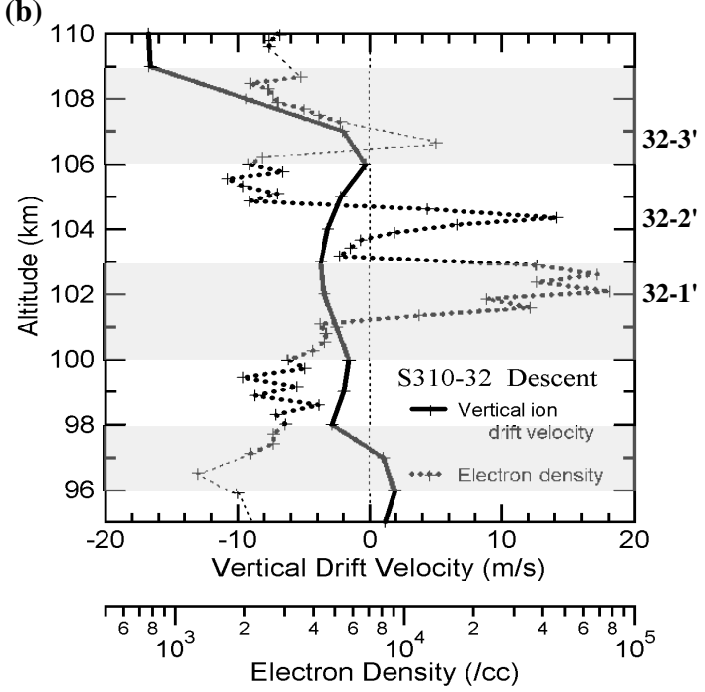

Fig. 2. The comparison between vertical ion drift velocities derived from neutral wind profiles and electron density profiles. The gray shadings represent the ion convergent regions due to the wind shear. The 32-2 and 32-2' peaks do not correspond to the ion accumulation regions in (a) and (b), respectively.

$106 \mathrm{~km}$ to $109 \mathrm{~km}$. Within the electron density profile of the descending phase, there were corresponding density peaks $32-1^{\prime}$ and $32-3^{\prime}$ at the altitudes of $102.1 \mathrm{~km}$ and $106.6 \mathrm{~km}$, respectively. The $32-2^{\prime}$ peak at $104.3 \mathrm{~km}$ did not correspondent to an ion accumulation region due to the wind shear.

\subsection{The effects of ionospheric electric field}

To understand the detailed dynamics of the $E_{S}$ ionospheric region, it is necessary to include the electric field effects. The data obtained by using the Electric Field Detector (EFD) (Pfaff et al., 2005) during the rocket flight makes it possible to examine the electric field effect.
To examine the $E_{s}$ layer's dynamics due to the electric fields, Eq. (1) is rewritten as follows,

$\frac{m_{i}}{e_{i}}\left(\boldsymbol{E}+\boldsymbol{V}_{i} \times \boldsymbol{B}\right)+v_{i n}\left(\boldsymbol{V}_{n}-\boldsymbol{V}_{i}\right)=0$.

Then, the $z$ component of the ion velocity is given as

$V_{z}=\frac{\alpha(z)}{\beta(z)}$,

where

$\alpha(z)=\frac{e_{i} E_{z}}{m_{i}}+\frac{e_{i} B_{y}}{m_{i}}\left\{\frac{\left(E_{x}+\frac{e_{i} E_{y} B_{z}}{m_{i} v_{i n}}+V_{n y} B_{z}\right) \frac{e_{i}}{v_{i n} m_{i}}+V_{n x}}{1+\frac{e_{i}^{2} B_{z}^{2}}{m_{i}^{2} v_{i n}^{2}}}\right\}$,

and

$\beta(z)=\frac{B_{y}^{2} e_{i}^{2} v_{i n}}{m_{i}^{2} v_{i n}^{2}+e_{i}^{2} B_{z}^{2}}+v_{i n}$.

Using Eq. (8), the vertical component of the ion drift velocity is plotted in Figs. 3a and b, and compared with the measured electron density profiles obtained by the S310-31 and S31032 rockets. To evaluate the effects of the electric fields and the neutral winds, the electric field data are averaged in each altitude range of $1 \mathrm{~km}$. In Figs. $3 \mathrm{a}$ and $\mathrm{b}$, three altitude regions are shaded in each panel where there is the possibility of ion convergence. In Fig. 3a (ascending phase), these regions are located from $101 \mathrm{~km}$ to $103 \mathrm{~km}$, from $104 \mathrm{~km}$ to $105 \mathrm{~km}$, and from $106 \mathrm{~km}$ to $108 \mathrm{~km}$. In Fig. 3a, the density peaks in the altitude range between $101 \mathrm{~km}$ and $103 \mathrm{~km} \mathrm{(31-}$ 1, 32-1) show a clear correspondence for both rocket flights. Moreover, in the S310-31 data as well, a clear density peak of 31-2 appears within the altitude region from $104 \mathrm{~km}$ to $105 \mathrm{~km}$. On the other hand, within the altitude range from $106 \mathrm{~km}$ to $108 \mathrm{~km}$, there is no correspondence in both profiles of the S310-31 and S310-32 ascending phases. The 31-3 peak appeared slightly higher $(100 \mathrm{~m})$ than the accumulation region. There are also three convergent altitude regions in Fig. $3 \mathrm{~b}$ (descending phase). These regions are from $97 \mathrm{~km}$ to $98 \mathrm{~km}$, from $99 \mathrm{~km}$ to $103 \mathrm{~km}$, and from $106 \mathrm{~km}$ to $109 \mathrm{~km}$. Within the altitude range from $97 \mathrm{~km}$ to $98 \mathrm{~km}$, there was not a significant peak in the measured density profile. In the region from $99 \mathrm{~km}$ to $103 \mathrm{~km}, 31-1^{\prime}$ and $32-1^{\prime}$ peaks were in agreement. In addition, the $31-3^{\prime}$ and $32-3^{\prime}$ peaks also corresponded to the region from $106 \mathrm{~km}$ to $109 \mathrm{~km}$ (the peak of the S310-31 is relatively small). However, the significant peak of $32-2^{\prime}$ in the descending phase is outside a convergent region, namely it is $1.3 \mathrm{~km}$ higher than the ion convergent region.

This shows that the electric field possibly causes the ions to converge into a layer at several altitude regions. The electric field effect appeared to cause a complex layer structure (for example, double peak $E_{S}(31-1,31-2)$ detected during the ascending phase of S310-31) although the 32-2' peak did not agree (this difference might be due to the difference in the observation region between S310-31 and S310-32 in the descending phase). Because a part of the electric field in the $E$-region ionosphere can be induced by the neutral wind, it 
is inferred that the induced electric field may reduce the ion accumulation. However, the results of this analysis suggest that the electric field intensifies the neutral wind effect and may form the complex structure observed during the SEEK2 campaign.

\subsection{DC electric field analysis}

It is suggested that the observed electric field has an important role in the formation of the complex structure of the $E_{s}$ layers. It is possible to estimate the magnitude of the polarization electric field due to the plasma density gradient and neutral wind under the tilted geomagnetic field. When a dense plasma region exists in the homogeneous background density, the relationship is given by the following equation,

$\mathbf{S}_{\mathbf{0}}\left(\boldsymbol{E}_{0}+\boldsymbol{U} \times \boldsymbol{B}\right)=\left(\mathbf{S}_{\mathbf{0}}+\mathbf{S}_{\mathbf{1}}\right)\left(\boldsymbol{E}_{0}+\boldsymbol{E}_{1}+\boldsymbol{U} \times \boldsymbol{B}\right)$,

where, $\mathbf{S}_{0}$ and $\mathbf{S}_{1}$ are the conductivity tensors of the background ionization region and dense plasma region, respectively. $\boldsymbol{E}_{0}$ and $\boldsymbol{E}_{1}$ are the initial and secondary electric field vectors, respectively. Neutral wind vectors $(\boldsymbol{U})$ are assumed to be horizontally homogeneous, and geomagnetic field vectors $(\boldsymbol{B})$ are also assumed to be homogeneous. The Pedersen, Hall and parallel conductivities are functions of ion and electron plasma frequency, cyclotron frequency, ion-neutral and electron-neutral collision frequency. Assuming that the ion species is $\mathrm{Fe}^{+}$, and charge neutrality, ion plasma frequencies are calculated by using the NEI observations. Collision frequencies can be deduced by using the relations,

$\omega_{i} \approx 1 \times 10^{2}[\mathrm{~Hz}]$,

and

$v_{\text {en }} \approx 6 \times 10^{-9} \cdot N_{n}$.

Where $N_{n}$ is the neutral density $\left(\mathrm{cm}^{-3}\right)$ given by the MSISE-90 atmospheric model. We assume the ionospheric background electron density is homogeneous, with $5 \times 10^{3} \mathrm{~cm}^{-3}$ (for the ascent period) and $3 \times 10^{3} \mathrm{~cm}^{-3}$ (for the descent period), based on the NEI observations for S310-31. In addition, the initial electric field $\boldsymbol{E}_{0}$ is also assumed to be zero. Neutral wind vectors are also obtained from the observation data by Larsen et al. (2005).

Equation (11) results are shown in Figs. $4 \mathrm{a}$ and $\mathrm{b}$ for ascent and descent of S310-31. In these four panels, calculated electric fields are over plotted and compared with the $E_{s}$ observations (Pfaff et al., 2005). As demonstrated by the descent profiles, the observed electric fields are displaced in the directions of south and west by several $\mathrm{mV} / \mathrm{m}$ to $5 \mathrm{mV} / \mathrm{m}$ from the calculated values. It can be interpreted that these displacements result from the addition of external electric fields in the downleg region. Pfaff et al. (2005) also suggested the existence of external electric fields. On the other hand, during the ascending period, the observed eastward electric fields were comparable with the calculated values. However, the $E_{s}$ peak altitudes always appeared in the region where the electric fields were shifted to the west and south directions. So, the electric fields directions played a role in the formation of the multiple $E_{s}$ layer structure.
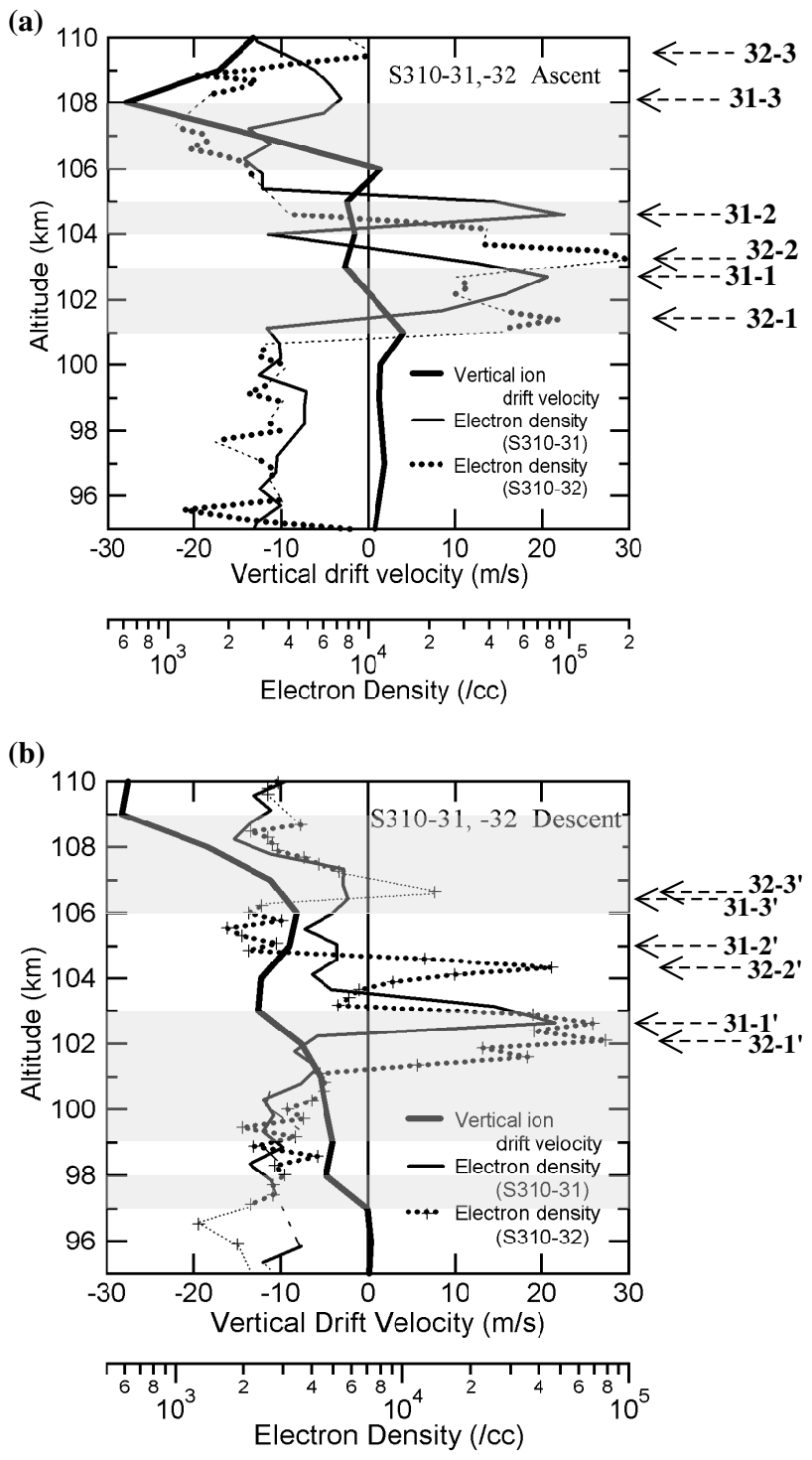

Fig. 3. Ion vertical drift velocity derived from neutral wind and electric field profiles obtained by S310-31 and S310-32 rockets.

\subsection{Contributions of wind and electric field}

In Figs. 5a and b, $V_{z}$ profiles, which were deduced from the wind measurement and observed electric field, are compared with the plasma density profiles measured by the S310-31 and S310-32 rockets. As was shown in Fig. 5, variation of vertical velocity based on the electric field is larger than that induced by the measured wind velocity. Thus, it seems plausible to assume that the contribution of the wind shear was stable, while that of the external electric field was variable during the SEEK-2 rocket experiment. In Figs. 5a and b, the peak of 31-1 is interpreted as the ion accumulation region, caused by wind, but peak 31-2 was generated by the external electric field alone. The peaks of $31-1^{\prime}$ and $31-3^{\prime}$ were in the accumulation region by the wind and the electric field. The 31-2' and 31-3 peaks did not correspond to the accumulation region (31-3 appeared at a slightly higher altitude 

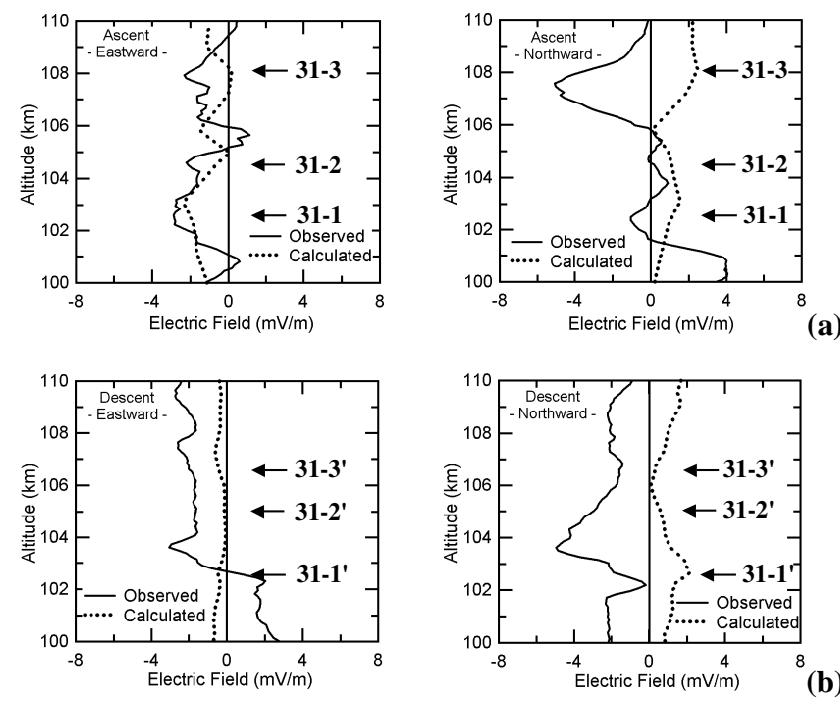

Fig. 4. Comparison between the observed electric fields and those calculated using Eq. (11). In the two panels (a), the observed and calculated profiles are similar in the eastward component though the northward component, disagrees especially around the 106-109 km altitude. The $E_{S}$ layers (detected by S310-31 during ascent) are in the altitude ranges where the electric fields are shifted to southwest. During the descending phase, panel (b), the electric fields are displaced in the direction of westward and southward, particularly above the altitude of $103 \mathrm{~km}$.

above the ion accumulation region caused by both wind and electric field). So, we can conclude that half of the $E_{s}$ layers of S310-31 were formed by the neutral wind effect only, or the combined effects of the neutral wind and the electric field. However, the upper peaks 31-2 of S310-31 can be interpreted as being only caused by the electric field.

\subsection{AC electric field observation}

As shown in Wakabayashi et al. (2005), intense VLF and ELF plasma waves appeared in the $E_{S}$ layer during the ascending and descending phases of S310-32. The strong plasma waves were detected within the time range from $114.99 \mathrm{~s}$ to $116.49 \mathrm{~s}$, which corresponds to the altitude range from $102.6 \mathrm{~km}$ to $103.3 \mathrm{~km}$ in the ascending phase. In the descending phase of the rocket, the strong plasma waves were also observed within $221.13 \mathrm{~s}$ to $221.72 \mathrm{~s}$ for the altitude range from $104.6 \mathrm{~km}$ to $104.3 \mathrm{~km}$. As shown in Fig. 6, these altitude ranges agree well with the upper peaks of the observed $E_{s}$ layers (32-2 and 32-2' peaks) during the ascending and descending phases. Therefore, these results imply an association between the plasma wave instability and the formation of the $E_{s}$ layers.

\section{Discussion}

The observations are summarized as follows:

1. From the electron density observations, different features among the observed $E_{s}$ layers in the multiple-layer (a)

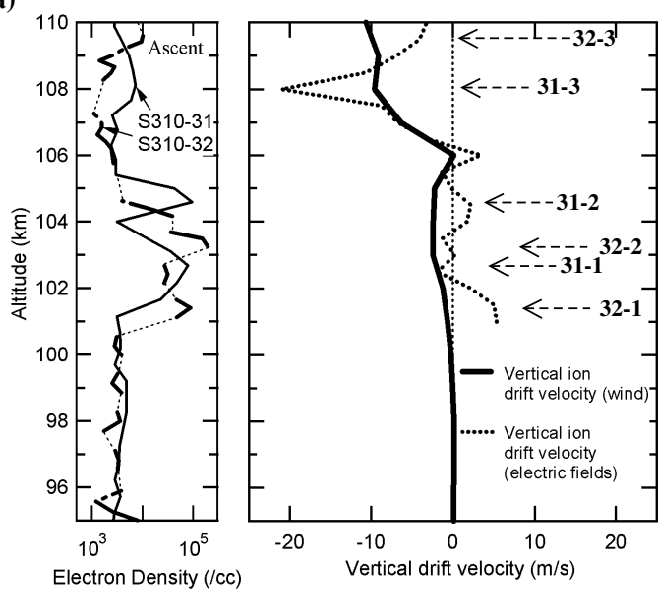

(b)

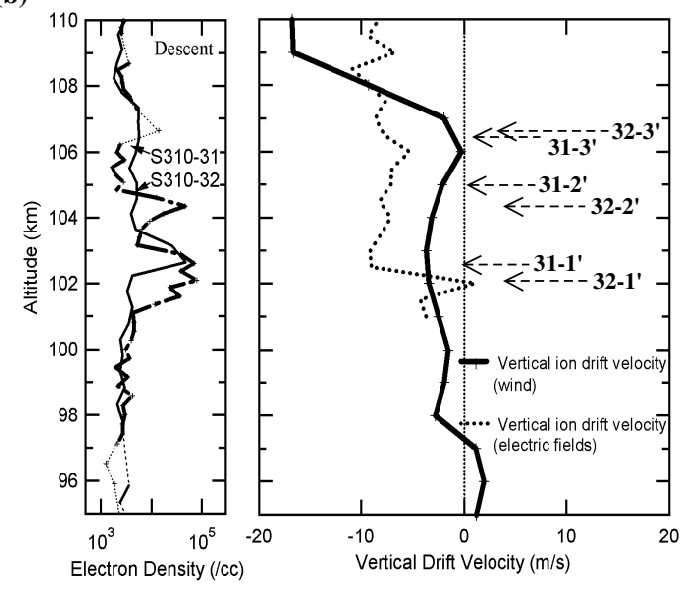

Fig. 5. (a) Comparison between the electron density profiles and ion vertical velocity. Here the ion vertical velocity is deduced from both the neutral wind (S310-32) and electric field data (S310-31). The peak $32-1$ is in the ion accumulation region by the wind and the electric field but peaks 31-2 and 32-2 are in the region due to the elctric field alone. The 31-3 peak is slightly above both accumulation regions. The peaks $31-1$ and $32-3$ are also in the regions due to netural wind accumulation. (b) The same as Fig. 5a, except for the descending phase. The $31-1^{\prime}, 31-3^{\prime}, 32-1^{\prime}$, and $32-3^{\prime}$ peaks are in the accumulation region due to neutral wind and the electric field. The $32-2^{\prime}$ is with the accumulation region due to the electric fields. The $31-2^{\prime}$ does not correspond to both accumulation regions.

structure were identified. The lower layers (peaks 31-1, $31-1^{\prime}, 32-1$ and $32-1^{\prime}$ ) tended to be relatively stable and broad, and the upper ones (31-2, 31-2', 32-2 and 32-2') tended to be unstable and narrow.

2. The lower layers corresponded to the ion accumulation regions due to the wind shear, but the upper layers did not. However, the upper layers also corresponded to the accumulation region due to the ionospheric electric field.

3. The observed DC electric fields include both external and induced internal electric fields. The magnitudes of 

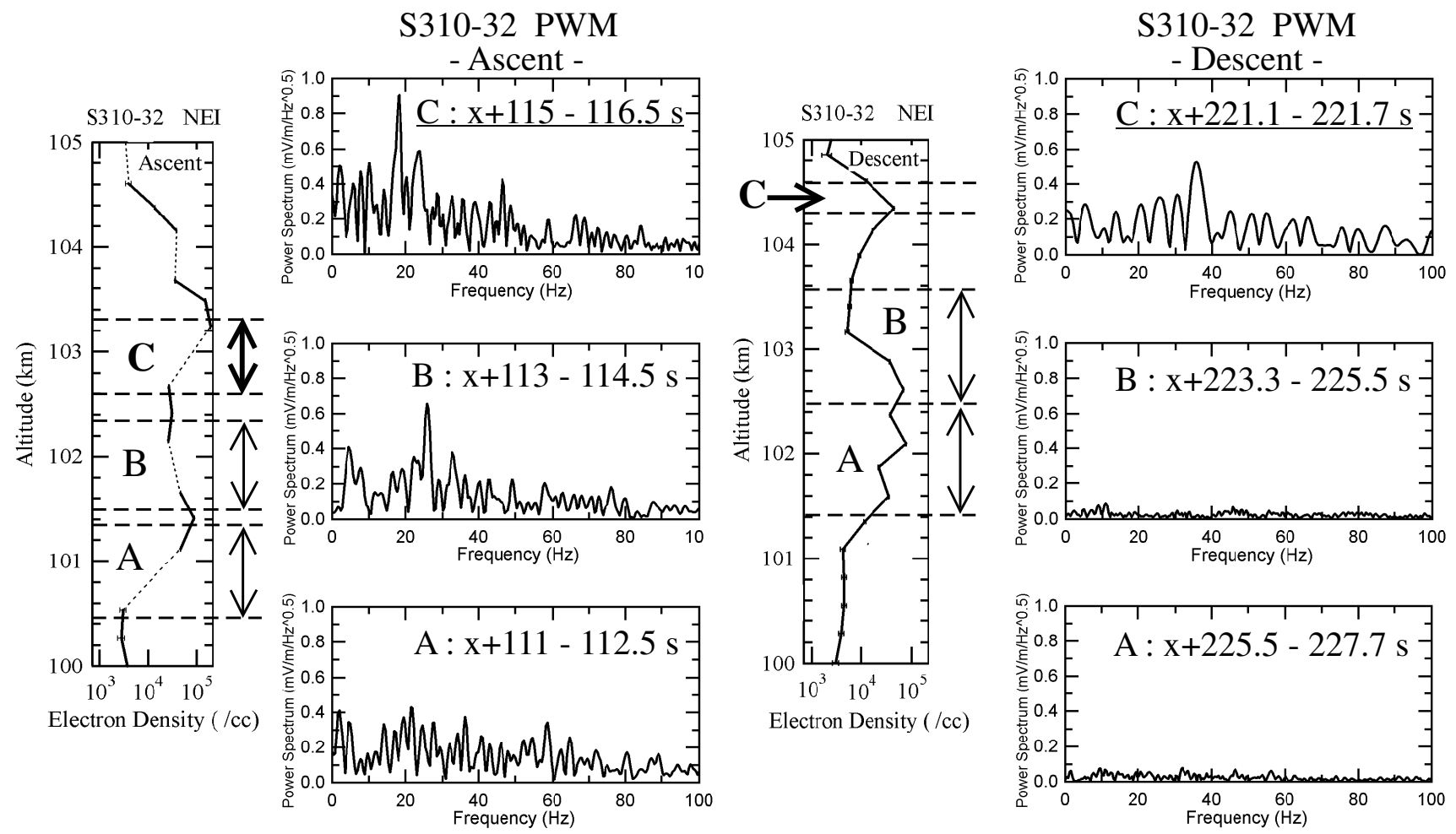

Fig. 6. The plasma wave spectra (FFT spectra) during passage through the sporadic- $E$. The left and right panels are in the ascending and descending phases of the S310-32 rocket.

external fields added to the downleg region were estimated to be several $\mathrm{mV} / \mathrm{m}$ to $5 \mathrm{mV} / \mathrm{m}$, directed to the southwest. The $E_{s}$ peak altitudes always existed within the altitude regions where the electric fields were displaced to the southwest direction in each profile.

4. The results of the AC electric field measurement (synchronized with the TMA release) showed that the electric field fluctuations were dominant in the upper layer $E_{s}$ region.

The achievement of the successive launches of two rockets during the $E_{s}$ layer event with the multi-layer structure made it possible to examine not only the shape but also the stability of each layer. In addition, the neutral wind and electric field measurements were simultaneously carried out to evaluate each contribution in the complex structure of the $E_{s}$ layer. We carried out data analysis for the relationship between the $E_{s}$ layer structure, the neutral wind, and the electric field of external origin. Pfaff et al. (2005) also pointed out the existence of northwest external electric fields, consistent with our results. Moreover, AC electric field measurement revealed a difference in the electrodynamic character between the upper and lower peaks. The TMA release experiment tends to interfere with other instruments due to the active chemical reaction. Thus, it is difficult to measure electric fields simultaneously with the TMA release. However, in this campaign we tried to measure the AC electric fields which were able to reveal electric field effects at the same time as the wind measurements.

Our analysis suggests that the generation mechanism of the upper layer was caused by the external electric field. The electric fields directions probably also contribute to the $E_{S}$ formation, as shown by the ascending phase measurements. It has been discussed that the electric field tends to form or stabilize the $E_{S}$ layers (Rees et al., 1976). This external electric field may originate in $F$-region disturbances.

During the SEEK-2 campaign, ionosonde observations were carried out in Yamagawa and Okinawa. Referring to the ionograms obtained at Okinawa, there were almost no disturbances in the $F$-region ionosphere. Although there were spread- $F$ signatures observed at Yamagawa, this disturbance did not map directly along the field lines to the $E$-region in the SEEK-2 observation region. The magnetic field lines on the SEEK-2 observation region maps to the $F$ region altitude (about $250 \mathrm{~km}$ ) around $29^{\circ} \mathrm{N}$ and $132.2^{\circ} \mathrm{E}$, which is almost half way between Okinawa and Yamagawa. If there were $F$-region disturbances around this area during the SEEK-2 campaign, it is possible to explain the origin of external electric fields. Perkins (1973) reported the $F$-region disturbances ("Perkins Instability") which can generate several $\mathrm{mV} / \mathrm{m}$ electric fields. It is plausible that such a plasma instability in the $F$-region ionosphere generated the external electric fields, and mapped to the $E$-region altitudes.

The numerical simulations of Machuga and Mathews (2001) described the ion trajectory in 3-D space, and 
it was suggested that the ion convergent altitude would shift a few km higher under the horizontally homogeneous electric field. However, the homogeneous electric field was in the direction of northwest. Although this electric field direction did not correspond to the present SEEK-2 campaign, the ion convergent altitude may have been shifted due to the external electric field. Therefore, the lower $E_{s}$ peak might be generated by the wind shear mechanism first, then the external electric field led to the formation of a higher $E_{S}$. The numerical simulations by Yokoyama et al. (2005; private communications) indicated the stability of the $E_{s}$ layer generated by only wind shears (although the actual $E_{s}$ layer has a "sporadic" nature). The present results are in agreement. Yokoyama et al. also reported that it was difficult to generate the sharp density gradient and high density in the $E_{s}$ layer due to the wind shear mechanism. It is interpreted that such as over dense layer (densities reaching $10^{5} \mathrm{~cm}^{-3}$ ) may be generated not only by ion convergence due to the wind shear and the electric field but also to some chemical process triggered by the external electric fields. Hence, the actual "sporadic" nature may be explained.

The previous explanation of multi-layer $E_{S}$ reported by Smith and Miller (1980) does not agree with the SEEK-2 results. The progress of this study is the indication of the difference between the lower and upper $E_{s}$ layers in the case of a multi-layer $E_{s}$ structure. This point has not been realized using previous observations made with sounding rocket and ISR instruments.

\section{Conclusions}

For the purpose of studying the QP echo phenomena associated with the $E_{s}$ layers, the comprehensive experiment of the SEEK-2 campaign was performed. The observed $E_{s}$ layer had the complex structure of dual peaks. The lower layer tended to be stable and of a slightly broad thickness, which was due to the wind shear mechanism. On the other hand, the upper layer was relatively unstable due to the electric field effects. This point was also suggested by the AC electric field measurement which was simultaneous with the TMA release.

The electric fields which contributed to the $E_{s}$ layer formation could be interpreted as external origin, and they may be generated by an $F$-region disturbance. The external fields may have existed during the whole SEEK-2 observation region, and its direction may also have contributed to the formation of unstable and stable layers.

Acknowledgements. The authors would like to give their hearty thanks to S. Fukao, M. Yamamoto of the Research Institute for Sustainable Humanosphere, Kyoto University, and to M. Iizima, A. Kumamoto of Tohoku University, for their important discussions and useful advice on the interpretation of rocket data.

The authors would like to express their deep appreciation to T. Yokoyama of the Solar-Terrestrial Environment Laboratory, Nagoya University, for his significant discussions and useful suggestions on the results of numerical simulations.
The authors wish to express their gratitude to the members of System-Keisoku Inc. for the development of the rocket-borne instruments and to all the members of the ISAS rocket team for the successful achievement of the SEEK-2 rocket experiments. Present work is supported by the 21 st century COE program "Advanced Science and Technology Center for the Dynamic Earth" at Tohoku University.

Topical Editor M. Pinnock thanks two referees for their help in evaluating this paper.

\section{References}

Axford, W. I.: The formation and vertical movement of dense ionized layers in the ionosphere due to neutral wind shears, J. Geophys. Res., 68, 769-779, 1963.

Bernhardt, P. A.: The modulation of sporadic-E layers by KelvinHelmholtz billows in the neutral atmosphere, J. Atmos. SolarTerr. Phys., 64, 1487-1504, 2002.

Bowen, P. J., Norman, K., Willmore, A. P., Baguette, J-M., Murtin F., and Storey L. R. O.: Rocket studies of sporadic- $E$ ionization and ionospheric winds, Planet. Space Sci., 12, 1173-1177, 1964.

Cosgrove, R. B. and Tsunoda, R. T.: Simulation of the nonlinear evolution of the sporadic- $E$ layer instability in the nighttime midlatitude ionosphere, J. Geophys. Res., 108(A7), 1283, doi:10.1029/2002JA009728, 2003.

Dorling E. B., Norman, K., and Willmore, A. P.: Rocket studies of sporadic- $E$ ionization, Planet. Space Sci., 17, 1207-1211, 1969.

Fujitaka, K. and Tohmatsu, T.: A tidal theory of the ionospheric intermediate layer, J. Atmos. Terr. Phys., 35, 3, 425-438, 1973.

Fukao, S., Yamamoto, M., Tsunoda, R. T., Hayakawa, H., and Mukai, T.: The SEEK (Sporadic- $E$ Experiment over Kyushu) campaign, Geophys. Res. Lett., 25, 1761-1764, 1998.

Haldoupis, C., Kelley, M. C., Hussey, G. C., and Shalimov, S.: Role of unstable sporadic- $E$ layers in the generation of midlatitude spread $F$, J. Geophys. Res., 108(A12), 1446, doi:10.1029/2003JA009956, 2003.

Huuskonen, A., Nygren, T., Jalonen, L., Bjørnå, N., Hansen, T. L., and Brekke, A.: Ion composition in sporadic $E$ layers measured by the EISCAT UHF radar, J. Geophys. Res., 93(A12), $14603-$ $14610,1988$.

Kato, S., Aso, T., Horiuchi, T., Nakamura, J., and Matsuoka, T.: Sporadic- $E$ formation by wind shear, comparison between observation and theory, Radio Sci., 7, 359-362, 1972.

Kirkwood, S.: Thin ion layers in the high-latitude lower ionosphere, Adv. Space Res., 19, 1, 149-158, 1997.

Kirkwood, S. and von Zahn, U.: On the role of auroral electric fields in the formation of low altitude sporadic- $E$ and sudden sodium layers, J. Atmos. Terr. Phys. 53, 5, 389-407, 1991.

Kirkwood, S. and von Zahn, U.: Formation mechanisms for lowaltitude $E_{S}$ and their relationship with neutral Fe layers: results from the METAL campaign, J. Geophys. Res., 98(A12), 21 549$21561,1993$.

Larsen, M. F., Yamamoto, M., Fukao, S., and Saito, A.: SEEK 2: Observations of neutral winds, wind shears, and wave structure during a sporadic-E/QP event, Ann. Geophys., 23, 2369-2375, 2005.

Machuga, D. W. and Mathews, J. D.: Numerical simulations of three-dimensional E-region ion trajectories in realistic tidal wind and E-field structures: layer formation and transport, J. Atmos. Solar-Terr. Phys., 63, 1519-1528, 2001. 
MacLeod, M. A.: Sporadic E Theory. I. Collision-Geomagnetic Equilibrium, J. Atmos. Sci., 23, 96-109, 1966.

Maruyama, T., Kato, H., and Nakamura, M.: Ionospheric effects of the Leonid meteor shower in November 2001 as observed by rapid run ionosondes, J. Geophys. Res., 108(A8), 1324, doi:10.1029/2003JA009831, 2003.

Mathews, J. D., Morton, Y. T., and Zhou, Q.: Observations of ion layer motions during the AIDA campaign, J. Atmos. Terr. Phys., 55, 3, 447-457, 1993.

Mathews, J. D., Machuga, D. W., and Zhou, Q.: Evidence for electrodynamic linkages between spread-F, ion rain, the intermediate layer, and sporadic E: results from observations and simulations, J. Atmos. Solar-Terr. Phys., 63, 1529-1543, 2001.

Nygren, T., Jalonen, L., Oksman, J., and Turunen, T.: The role of electric field and neutral wind direction in the formation of sporadic E-layers, J. Atmos. Terr. Phys., 46, 4, 373-381, 1984.

Ogawa, T., Takahashi, O., Otsuka, Y., Nozaki, K., Yamamoto, M., and Kita, K.: Simultaneous middle and upper atmosphere radar and ionospheric sounder observations of midlatitude $E$ region irregularities and sporadic $E$ layer, J. Geophys. Res., 107(A10), 1275, doi:10.1029/2001JA900176, 2002.

Perkins, F.: Spread $F$ and ionospheric currents, J. Geophys. Res., 78, 1, 218-226, 1973.

Pfaff, R., Freudenreich, H., Yokoyama, T., Yamamoto, M., Fukao, S., Mori, H., Ohtsuki, S., and Iwagami, N.: Electric field measurements of DC and long wavelength structures associated with sporadic- $E$ layers and QP radar echoes, Ann. Geophys., 23, 2319-2334, 2005.

Rees, D., Dorling, E. B., Lloyd, K. H., and Low, C.: The role of neutral winds and ionospheric electric field in forming stable sporadic E-layers, Planet. Space Sci., 24, 475-478, 1976.
Roddy, P. A., Earle, G. D., Swenson, C. M., Carlson, C. G., and Bullett, T. W.: Relative concentrations of molecular and metallic ions in midlatitude intermediate and sporadic-E layers, Geophys. Res. Lett., 31, L19807, doi:1029/2004GL020604, 2004.

Smith, L. B. and Wright, J. W.: Sporadic- $E$ and wind-profile interrelation over Hawaii, Radio Sci., 7, 3, 363-366, 1972.

Smith, L. G.: Rocket observations of sporadic $E$ and related features of the $E$ region, Radio Sci., 1, 178-186, 1966.

Smith, L. G. and Mechtly, E. A.: Rocket observations of sporadic- $E$ layers, Radio Sci., 7, 367-376, 1972.

Smith, L. G. and Miller, K. L.: Sporadic- $E$ layers and unstable wind shears, J. Atmos. Terr. Phys., 42, 45-50, 1980.

Wakabayashi, M., Ono, T., Mori, H., and Bernhardt, P. A.: Electron density and plasma waves in mid-latitude sporadic- $E$ layer observed during the SEEK-2 campaign, Ann. Geophys., 23, 23352345, 2005.

Whitehead, J. D.: The formation of the sporadic- $E$ layer in the temperate zones, J. Atmos. Terr. Phys., 20, 49-58, 1961.

Yamamoto, M., Fukao, S., Tsunoda, R. T., and Hayakawa, H.: SEEK-2 (Sporadic- $E$ Experiment over Kyushu 2) - Project Outline and Significance, Ann. Geophys., 23, 2295-2305, 2005.

Yamamoto, M.-Y., Ono, T., Oya, H., Tsunoda, R. T., Larsen, M. F., Fukao, S., and Yamamoto, M.: Structures in sporadic- $E$ observed with an impedance probe during the SEEK campaign : Comparisons with neutral-wind and radar-echo observations, Geophys. Res. Lett., 25, 1781-1784, 1998.

Yokoyama, T., Yamamoto, M., Fukao, S., Takahashi, T., and Tanaka, M.: Numerical simulation of midlatitude ionospheric $E$ region based on the SEEK and the SEEK-2 observations, Ann. Geophys., 23, 2377-2384, 2005. 